\title{
Radial endobronchial ultrasound-assisted transbronchial needle aspiration for pulmonary peripheral lesions in the segmental bronchi adjacent to the central airway
}

\author{
Nan Song ${ }^{1,2 \#}$, Li Yang ${ }^{3 \#}$, Hao Wang ${ }^{2,4 \#}$, Lei Jiang ${ }^{1,2 \#}$, Lishu Zhao ${ }^{2,4}$, Sara Colella ${ }^{5}$, Nikhil Jagan ${ }^{6}$, \\ Francisco A. Almeida ${ }^{7}$, Liang $\mathrm{Wu}^{8}$, $\mathrm{Ye} \mathrm{Gu}^{3}$, Yayi $\mathrm{He}^{2,4}$ \\ ${ }^{1}$ Department of Thoracic Surgery, Shanghai Pulmonary Hospital, Tongji University School of Medicine, Shanghai, China; ${ }^{2}$ School of Medicine, \\ Tongji University, Shanghai, China; ${ }^{3}$ Department of Endoscopy Center, Shanghai Pulmonary Hospital, Tongji University School of Medicine, \\ Shanghai, China; ${ }^{4}$ Department of Medical Oncology, Shanghai Pulmonary Hospital, Tongji University Medical School Cancer Institute, Tongji \\ University School of Medicine, Shanghai, China; ${ }^{5}$ Pulmonary Unit, “G. Mazzini” Hospital, Teramo, Italy; ${ }^{6}$ Division of Pulmonary, Critical Care, \\ and Sleep Medicine, Creighton University Medical Center, Omaha, NE, USA; ${ }^{7}$ Respiratory Institute, Cleveland Clinic, Cleveland, OH, USA; \\ ${ }^{8}$ Department of Thoracic Surgery, Shanghai General Hospital, Shanghai Jiao Tong University School of Medicine, Shanghai, China \\ Contributions: (I) Conception and design: Y Gu, Y He; (II) Administrative support: Y Gu; (III) Provision of study materials or patients: L Yang, Y Gu; \\ (IV) Collection and assembly of data: N Song, L Yang, L Zhao; (V) Data analysis and interpretation: L Jiang, N Song, H Wang; (VI) Manuscript \\ writing: All authors; (VII) Final approval of manuscript: All authors. \\ "These authors contributed equally to this work as co-first authors. \\ Correspondence to: Yayi He. No 507 Zhengmin Road, Shanghai 200433, China. Email: doctorjael@qq.com; Ye Gu. No 507 Zhengmin Road, Shanghai \\ 200433, China. Email: Drsymons@outlook.com; Liang Wu. No. 100 Haining Road, Shanghai 200080, China. Email: wuliang198209@yahoo.com.
}

Background: Tissue samples from lesions located in the 3 rd to 5 th segmental bronchi are challenging to obtain. In this retrospective study, we aimed to evaluate the diagnostic rate of pulmonary peripheral lesions located in the 3 rd to 5 th segmental bronchi, near the inner field of lung on the computed tomography (CT) image and outside the bronchus, using radial endobronchial ultrasound (REBUS) followed by transbronchial needle aspiration (TBNA).

Methods: This retrospective study enrolled patients whose preoperative CT examinations showed a lesion located in the segmental bronchi (3rd to 5th), yet adjacent to the inner field of lung on the CT image. REBUS followed by TBNA was used to acquire tissue samples from these lesions. A bronchoscope was used to reach the bronchi surrounding the lesion, and an ultrasound probe was used to determine the lesion's location. Then, the ultrasound probe was withdrawn, and puncture was performed at the location that was determined by ultrasound. The tissue specimens obtained were subjected to pathological examination.

Results: Nineteen patients were enrolled in this study including 15 males and 4 females with an average age of 55 years old. Of the enrollees, 8 patients (42.1\%) were successfully diagnosed with samples obtained through TBNA, including 6 cases of lung cancer, 1 case of non-specific inflammation, and 1 case of cryptococcal infection. The diagnostic rate was $42.1 \%$. No post-procedural complications were observed among the patients. There was no significant difference in nodule diameter between patients with a diagnostic sample and those in whom TBNA failed to provide a diagnosis $(2.99 \pm 0.96$ vs. $2.26 \pm 1.27 \mathrm{~cm}$, $\mathrm{P}=0.20)$.

Conclusions: With the assistance of REBUS, TBNA can acquire sufficient samples to achieve a reasonably diagnostic rate for parenchymal lung lesions located near the inner field of lung on the CT image without intrabronchial invasion.

Keywords: Radial endobronchial ultrasound (REBUS); transbronchial needle aspiration (TBNA); segmental bronchi; diagnosis 
Submitted Mar 31, 2021. Accepted for publication Jun 23, 2021.

doi: $10.21037 /$ tlcr-21-490

View this article at: https://dx.doi.org/10.21037/tlcr-21-490

\section{Introduction}

Lung cancer ranks first out of all malignancies for morbidity and mortality in most countries $(1,2)$. Early diagnosis and treatment are key to curing lung cancer and prolonging survival. The American National Lung Screening Trial (NCT00047385) demonstrated that screening with lowdose computed tomography (CT) lead to a relative reduction in mortality of $20 \%$ among patients with lung cancer (3). Consequently, this screening method has been accepted by the academic community and included in clinical guidelines. With large-scale CT screening, an increasing number of unidentified lung lesions appears to be occurring $(4,5)$. Therefore, the optimal diagnostic approach for patients with suspicious nodules urgently needs to be determined.

Malignant diseases cannot be accurately distinguished from benign conditions by noninvasive examination methods, such as fluorodeoxyglucose positron emission tomography or dynamic contrast-enhanced CT (6). For such indeterminate lung nodules, many invasive methods for obtaining tissue exist. Probably the most widely available, CT-guided lung biopsy is associated with a high incidence of complications (up to $40 \%$ ), with the incidence of pneumothorax reaching up to $25 \%(7,8)$.

Many minimally invasive flexible bronchoscopic procedures are currently available. These include convex probe endobronchial ultrasound (CP-EBUS), radial endobronchial ultrasound (REBUS), virtual bronchoscopy (VB), and electromagnetic navigation bronchoscopy (ENB). However, the availability of ENB and VB remains extremely limited in many parts of the world, mostly due to the elevated costs of these procedures. The access to these lesions in the vicinity of the $3 \mathrm{rd}$ to 5 th segmental bronchi by CP-EBUS may be limited by the scope's large size $(6.9 \mathrm{~mm}$ in diameter). Finally, when no endobronchial component or extrinsic compression is present, adequate sampling is generally limited to transbronchial needle aspiration (TBNA). TBNA has been widely used in diagnosing both benign and malignant pulmonary diseases. EBUS is a good approach for visualizing lesions to guide the sample biopsy and EBUS-TBNA has been suggested to have a sensitivity around 0.9 and a specificity to 1.0 in lung cancer diagnosing and staging $(9,10)$. Currently, REBUS has been developed as a novel technique to guide TBNA sampling (11). In this retrospective study, we aimed to evaluate the diagnostic rate and complications when using REBUS followed by TBNA in the evaluation of lesions located in the 3 rd to 5 th segmental bronchi, near the inner lung field on the CT image and outside the bronchus. We present the following article in accordance with the STROBE reporting checklist (available at https://dx.doi.org/10.21037/tlcr-21-490).

\section{Methods}

\section{Study participants}

Patients who underwent REBUS-TBNA in Shanghai Pulmonary Hospital between August 2016 and May 2020 were included in this retrospective study. During this time, 900 patients underwent REBUS in the hospital. Because of the specific location of the lesion, nineteen out of 900 patients conformed to the inclusion criteria (Figure S1). Clinical and pathological parameters including the patient's age, sex, smoking history, the lesion location and the lesion size were recorded from patients' medical records and medical images.

The inclusion criteria for patients were: (I) aged 18 to 80 years old; (II) clinically suspected lung lesion located in the segmental bronchi ( 3 rd to 5 th) in addition to its location near the inner lung field on the CT image; (III) no contraindications to bronchoscopy; (IV) signed an informed consent form and approval for medical record reviewing. The study received approval from the Ethics Committee of Tongji University Affiliated Shanghai Pulmonary Hospital (No. 18Q016NJ) in accordance with the guidelines of the Helsinki Declaration (as revised in 2013). Written informed consents were obtained from all patients for reviewing their medical records and images for scientific research.

\section{REBUS-TBNA}

Preoperative investigations included an enhanced CT scan of the lungs, complete blood count (CBC), liver and renal function examination, electrolytes examination, electrocardiogram (ECG), and coagulation tests. 

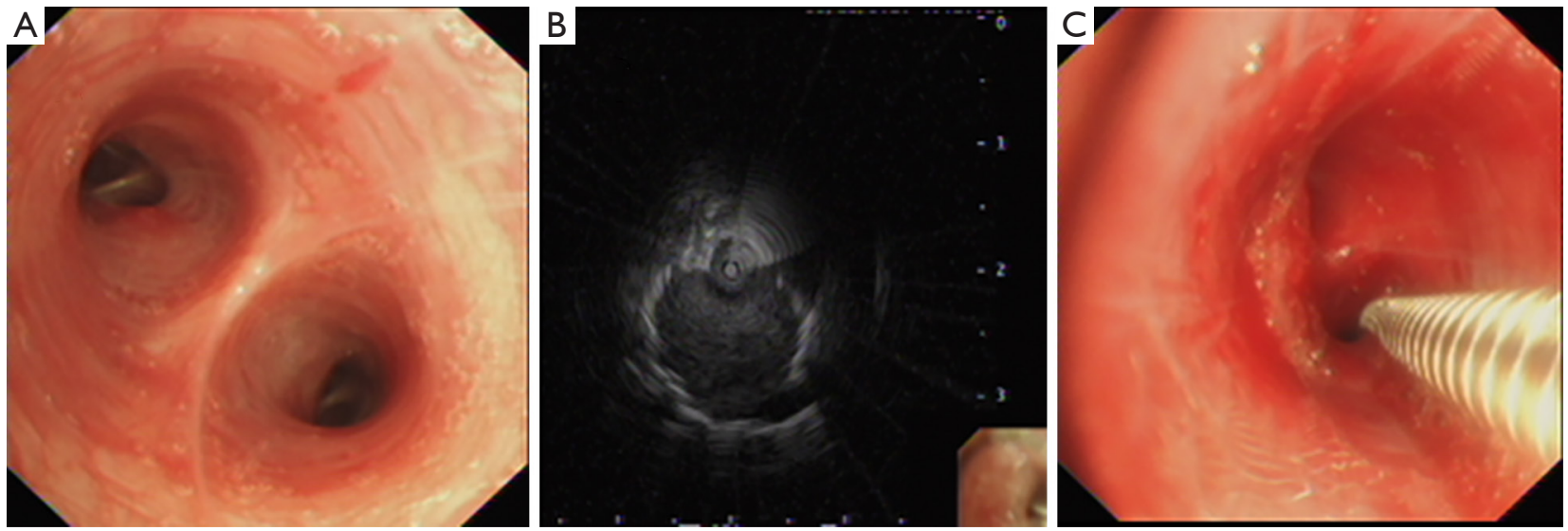

Figure 1 The representative images during REBUS-TBNA procedure. (A) Bronchoscopic image of the right middle lobe bronchus of a patient; (B) radial ultrasound image of a lesion in the right middle lobe; (C) puncture of the right middle lobe lesion. REBUS, radial endobronchial ultrasound; TBNA, transbronchial needle aspiration.

Immediately before the procedure, the operator reviewed the chest CT images and determined the lesions size and the bronchus in which was located. The patient was instructed to lie on the examination table in the supine position and received nebulized lidocaine (administered oropharyngeally) as local anesthesia. Then oxygen was provided via nasal cannula for the entire length of the procedure and monitored for vital signs. In our current cohort intravenous sedation or general anesthesia was not applied.

The procedure was performed with the Olympus BFP260F bronchoscope (Olympus Co. Ltd., Tokyo, Japan). After a systematic inspection of the tracheobronchial tree (Figure 1A), the REBUS (UM-S20-20R, Olympus Co. Ltd., Tokyo, Japan) $20-\mathrm{MHz}$ probe was inserted into the target bronchus on the bases of the CT-scan images as discussed above. Once the best possible ultrasound image of the peribronchial lesion was obtained with the REBUS probe (Figure 1B), the segmental bronchus was recorded, and the optimal puncture location was selected. Before the withdrawal of the ultrasound probe, the probe depth was marked. Subsequently, a 19-gauge WANG TBNA needle (MWF-319, ConMed Company, New York, USA) was inserted through the biopsy channel at the same length of the mark done on the REBUS probe, and a needle biopsy was performed at the location that was selected under ultrasound image (Figure 1C). Then, a $60-\mathrm{mL}$ syringe was attached to the end of the needle with negative pressure aspiration applied. The process was repeated to obtain 5 to 6 samples. Brush cytology was performed routinely to avoid missing any mucosal involvement by the lesion not macroscopically identified on the airway exam. Biopsy tissues would be sent for histopathological examination and smear cytological examination. If an accurate diagnosis could be obtained from the biopsy sample, the sample would be considered as a diagnostic sample and the patient would be defined as patient with positive diagnosis.

The specimens were fixed in a $10 \%$ formalin solution for histopathological examination. The molecular testing was polymerase chain reaction (PCR)-based assay. DNA or RNA would be extracted from biopsy samples according to the protocol. Reverse transcription would be done to convert RNA into cDNA for further PCR. The aberrations of EGFR/ALK/ROS1/KRAS/BRAF were detected by MultiGene Mutations Detection Kit (Amoy, Xiamen, China) according to manufacturer's protocol.

After the procedure, if bleeding occurred, local hemostasis was carried out using diluted epinephrine and cold saline. After confirmation of no active bleeding, the procedure was completed. Intraoperative and postoperative adverse events were recorded including bleeding, chest pain, hypoxia, and postoperative infection. All procedures were performed by the same bronchoscopist, who was aided by the same nurse, to avoid the potential bias.

\section{Statistical analyses}

Data were statistically analyzed with IBM SPSS Statistics 22 (IBM, Armonk, NY, USA). Continuous variables were expressed as mean \pm standard deviation, and categorical variables as counts and percentages. Fisher's exact test was 
Table 1 Clinical characteristics of patients with lung lesions

\begin{tabular}{|c|c|c|c|c|c|c|c|}
\hline Patient No. & Sex & Age (years) & Smoker & Lesion diameter (cm) & Lesion location & Pathology & Diagnosis \\
\hline 2 & M & 52 & No & 1.3 & RLL, B6 & ND & $\mathrm{N}$ \\
\hline 3 & $\mathrm{~F}$ & 45 & No & 3.1 & LUL, B5 & Inflammation & $\mathrm{P}$ \\
\hline 4 & M & 56 & No & 3.8 & RUL, B2b & Cryptococcal infection & $\mathrm{P}$ \\
\hline 6 & M & 33 & No & 2.5 & RLL, B6 & ND & $\mathrm{N}$ \\
\hline 7 & M & 48 & No & 2.5 & RLL, B8 & ND & $\mathrm{N}$ \\
\hline 8 & M & 52 & No & 4.0 & LUL, B3 & ND & $\mathrm{N}$ \\
\hline 9 & M & 60 & Yes & 1.2 & LUL, B1 & ND & $\mathrm{N}$ \\
\hline 12 & $\mathrm{~F}$ & 65 & No & 1.9 & LLL, B10 & ND & $\mathrm{N}$ \\
\hline 13 & M & 53 & Yes & 3.9 & LUL, B3 & Carcinoma & $\mathrm{P}$ \\
\hline 14 & $\mathrm{~F}$ & 56 & No & 2.7 & RML, B4 & ND & $\mathrm{N}$ \\
\hline 15 & $\mathrm{~F}$ & 25 & No & 0.8 & RUL, B2 & ND & $\mathrm{N}$ \\
\hline 16 & M & 65 & No & 4.3 & LUL, B1 & Adenocarcinoma & $\mathrm{P}$ \\
\hline 17 & M & 67 & No & 2.8 & RUL, B1 & ND & $\mathrm{N}$ \\
\hline 18 & M & 69 & No & 1.7 & RUL, B2 & Adenocarcinoma & $\mathrm{P}$ \\
\hline 19 & M & 66 & No & 2.9 & RLL, B8 & Small cell lung cancer & $\mathrm{P}$ \\
\hline
\end{tabular}

M, male; F, female; RUL, right upper lobe; RML, right middle lobe; RLL, right lower lobe; LUL, left upper lobe; LLL, left lower lobe; ND, non-diagnostic; $\mathrm{P}$, positive diagnosis; $\mathrm{N}$, negative diagnosis.

used to compare the distributions of sex, age, smoking history, and nodule diameters between groups. Nodule diameters for patients with different diagnoses ("positive" or "negative") were compared using the $t$-test. Statistical significance was indicated by a $\mathrm{P}$ value of less than 0.05 . The diagnostic yield was calculated by dividing the number of successful diagnoses by the total number of lesions.

\section{Results}

This study included 19 patients [15 males (78.9\%)], with an average age of $55( \pm 12.1)$ years old (Table 1). All patients had their reports of pathological examination and were included for further analysis. Eight nodules were located in the left lung, of which only one was in the left lower lobe. Of the eleven right lung nodules, six were located in the right upper lobe, one in the right middle lobe and four in the right lower lobe (Table 1). Lesion size was obtained from the CT images and the average diameter of all lesions was $2.57 \pm 1.18 \mathrm{~cm}$ (Table 1). No bronchus sign was observed on CT images in any patients. All lung nodules were visualized under REBUS during the procedure.

In total, 8 patients $(42.1 \%)$ were successfully diagnosed with TBNA samples and most of these were malignant (Table 1). Six cases were ultimately diagnosed with lung cancer, one with cryptococcal infection, and one with nonspecific inflammation. Two brush samples $(10.5 \%)$ were diagnostic and showed lung adenocarcinoma. The overall diagnostic rate was $42.1 \%$ [8/19]. Among the patients with a positive diagnosis, 3 lesions were in the left upper lobe, 4 lesions in the right upper lobe, and 1 lesion in the right lower lobe. Meanwhile, in the 6 samples diagnosed as lung cancer, 3 samples were also suitable for detecting driver gene alteration (EGFR/ALK/BRAF/KRAS/ROS1). One of these was found to have $B R A F$ V600E mutation and no driver gene alteration was detected in the other two. Among 
Table 2 Comparison of clinical parameters according to diagnostic status (positive or negative)

\begin{tabular}{|c|c|c|c|c|}
\hline Group & Positive & Negative & Total & $P$ value \\
\hline Male & 7 & 8 & $15(78.9)$ & \\
\hline Female & 1 & 3 & $4(21.1)$ & \\
\hline Age (years) & & & & 1.00 \\
\hline$>55$ & 4 & 6 & $10(52.6)$ & \\
\hline Smoking history & & & & 1.00 \\
\hline Non-smoker & 6 & 9 & 15 (78.9) & \\
\hline Smoker & 2 & 2 & $4(21.1)$ & \\
\hline$>2.5$ & 5 & 4 & $9(47.4)$ & \\
\hline
\end{tabular}

patients with a negative diagnosis, 1 lesion was in left lower lobe, 4 lesions in left upper lobe, 3 lesions in right lower lobe, 1 lesion in right middle lobe and 2 lesions in right upper lobe.

No significant difference was found in nodule diameter between patients with a positive and negative diagnoses (2.99 \pm 0.96 vs. $2.26 \pm 1.27 \mathrm{~cm}, \mathrm{P}=0.20)$. Other clinical characteristics were also similar between diagnostic and non-diagnostic samples $(\mathrm{P}>0.05$, Table 2). Furthermore, no postoperative complication was recorded among the study participants.

\section{Discussion}

The present study included 19 patients with peribronchial lesions located in the vicinity of the $3 \mathrm{rd}$ to 5 th segmental bronchi, near the inner field of the lung on the CT image and outside the airway lumen. Obtaining tissue samples from such lesions can be challenging. The airway diameter may not be large enough to be able to fit a CP-EBUS for real-time sampling. Also, as the lesions are often completely outside the airway, endobronchial forceps biopsy and brush techniques are not good options. As a result, conventional TBNA (C-TBNA) may turn out to be the only available tool for obtaining pathological tissue samples in many of these cases (11). C-TBNA was first invented by Dr. Eduardo Schieppati in 1949 and was further developed by Ko-Pen Wang (12). It has been applied successfully in the clinical setting for more than 20 years. With the guidance of REBUS described herein, C-TBNA remains a valuable tool.

In the current study, the diagnostic yield of REBUS for nodules with an average diameter of $2.57 \mathrm{~cm}$ near the inner field of the lung was $42.1 \%$. A retrospective analysis of 177 patients revealed diagnostic rates of $14 \%$ and $31 \%$ for lesions of $<2 \mathrm{~cm}$ located in the peripheral third and the inner two-thirds of the lung, respectively (13), which almost corresponded with our results. Ost and colleagues have described the diagnostic yield of REBUS to be $57.0 \%$ with a higher diagnostic yield for lesions greater than $2 \mathrm{~cm}$ in longest diameter (14). A prospective trial with 54 patients with nodules that could not be visualized by fluoroscopy reported 48 lesions (89\%) were localized by REUBS, and in 38 cases $(70 \%)$ diagnoses were established by biopsy. One pneumothorax occurred in this series (15). In another study, the diagnostic yield of REBUS was 70.6\% (16), which was superior to that previously reported for routine bronchoscopy $(13,17)$. Wang Memoli et al. also reported the diagnostic yield of REBUS to be $71.1 \%$ in a meta-analysis (18). Moreover, the currently reported diagnostic yield of REBUS was comparable to that reported by Gildea $e t a l$. for ENB (74\%) (19) and Asano et al. for VB (67.1\%) (20). It suggests that REBUS-TBNA is an efficient and costeffective diagnostic method. In our study, we only included nodules located in the inner field of the lung. The prevalence of such lesions in previous studies is not well 
described. Thus, the relatively low diagnostic yield in our study might be related to the very specific location of the lesions. Further studies are indispensable to evaluate the diagnostic performance of REBUS for lesions at different segmental bronchi. Meanwhile, according to our current study, the sample obtained from REBUS-TBNA may also suitable for molecular testing, which is corresponding to previous study (21).

The diagnostic sensitivity of trans-bronchoscopic biopsy for peripheral lung cancer has been shown to be related to lesion size $(13,22)$. With traditional EBUS, the larger the lesion, the greater the likelihood of obtaining a diagnostic sample (23). A recent meta-analysis of 57 studies indicated that the diagnostic rate was significantly higher for lesions with a diameter of $>2 \mathrm{~cm}$ (16). In this study, we found that the nodule size did not differ significantly between patients with diagnostic and non-diagnostic samples. However, because of the small sample size of our study, these findings need to be interpreted with caution and larger studies are needed.

In a study of 846 CT-guided percutaneous procedures, the mean lesion size was $3.02 \mathrm{~cm}$ and the complications rate was $30 \%$, with the incidence of pneumothorax and hemoptysis reaching $27 \%$ and $3 \%$, respectively (24). The risk of pneumothorax is 11 times higher in patients with lesions $<2 \mathrm{~cm}$ than in patients with lesions $>4 \mathrm{~cm} \mathrm{(25).}$ The rate of pneumothorax during routine bronchoscopy is reported to be $4 \%(26,27)$. The risk of complications from REBUS is quite low, with an approximate risk of pneumothorax of only $1 \%$ (28), which was in line with our research findings. Furthermore, the incidence of adverse events requiring intervention is only $0.7 \%$ (18).

Although REBUS-TBNA can improve the diagnosis of those lesions located in 3rd-5 th segmental bronchus, it has certain shortcomings. For instance, radial ultrasound is not self-navigating or localizing, and its path of exploration depends on the operator's individual understanding of lesion localization. Previous studies reported that approximately $25.0 \%$ to $33.3 \%$ of lesions were undefined $(29,30)$.

Our study also had some limitations. First, the small sample size, the single-center and retrospective nature of the present study. Second, the patient without identical lesion under REBUS was not included in the study. Third, due to the lack of long-term follow-up, true sensitivities could not be calculated. Also, there was a higher number of non-smokers in our cohort which might suggest a larger than usual number of benign etiologies. This could have impacted our diagnostic yield as a TBNA technique is not as sensitive for benign disease as it is to malignant lesions.
Finally, sampling of these nodules with CP-EBUS was not attempted. It has been shown that some of these lesions can be sampled with CP-EBUS even when the nearby airway diameter is smaller than the scope (31). Perhaps the CPEBUS scope dilates the airway to a certain extent. So, it is possible REBUS and CP-EBUS may be complementary for lesions such as the ones described in our report. Especially for upper lobe lesions where the currently available CPEBUS does not perform well.

\section{Conclusions}

REBUS-TBNA is a minimally invasive biopsy technique with a reasonable diagnostic rate for more central lesions and a low incidence of complications. TBNA can be used with new guided instrumentation, and more flexible dedicated peripheral tools may further improve diagnostic rates. In addition, REBUS-TBNA has emerged in the field of tumor precision therapy and it may eventually play a significant role in accurate concomitant tumor detection, typing, and treatment $(21,32,33)$. Our future work will continue to explore and improve the clinical value of REBUS-TBNA.

\section{Acknowledgments}

The authors appreciate the academic support from AME Lung Cancer Collaborative Group.

Funding: This study was supported in part by a grant of National Natural Science Foundation of China (81802255), Clinical research project of Shanghai Pulmonary Hospital (FKLY20010), Young Talents in Shanghai (2019 QNBJ), “Dream Tutor" Outstanding Young Talents Program (fkyq1901), Clinical Research Project of Shanghai Pulmonary Hospital (FKLY20001), Respiratory Medicine, a key clinical specialty construction project in Shanghai, Promotion and application of multidisciplinary collaboration system for pulmonary non-infectious diseases, Clinical Research Project of Shanghai Pulmonary Hospital (fk18005), Key Discipline in 2019 (oncology), Project of Shanghai Municipal Science and Technology Commission (Project of Municipal Science and Technology Commission), Scientific research project of Shanghai Pulmonary Hospital (fkcx1903), Shanghai Municipal Commission of Health and Family Planning (2017YQ050), Innovation Training Project of SITP of Tongji University, and key projects of leading talent (19411950300). Youth project of hospital management research fund of Shanghai 
Hospital Association (Q1902037), the grant from China National Key Research and Development Plan Project (2017YFC0113501).

\section{Footnote}

Reporting Checklist: The authors have completed the STROBE checklist. Available at https://dx.doi. org/10.21037/tlcr-21-490

Data Sharing Statement: Available at https://dx.doi. org/10.21037/tlcr-21-490

Conflicts of Interest: All authors have completed the ICMJE uniform disclosure form (available at https://dx.doi. org/10.21037/tlcr-21-490). YH serves as an unpaid editorial board member of Translational Lung Cancer Research from Jan 2020 to Dec 2021. The other authors have no conflicts of interest to declare.

Ethical Statement: The authors are accountable for all aspects of the work in ensuring that questions related to the accuracy or integrity of any part of the work are appropriately investigated and resolved. The study was conducted in accordance with the Declaration of Helsinki (as revised in 2013). The study was approved by Ethics Committee of Tongji University Affiliated Shanghai Pulmonary Hospital (No. 18Q016NJ) and individual consent for this retrospective analysis had been obtained from all participants.

Open Access Statement: This is an Open Access article distributed in accordance with the Creative Commons Attribution-NonCommercial-NoDerivs 4.0 International License (CC BY-NC-ND 4.0), which permits the noncommercial replication and distribution of the article with the strict proviso that no changes or edits are made and the original work is properly cited (including links to both the formal publication through the relevant DOI and the license). See: https://creativecommons.org/licenses/by-nc-nd/4.0/.

\section{References}

1. Siegel RL, Miller KD, Jemal A. Cancer statistics, 2020. CA Cancer J Clin 2020;70:7-30.

2. He Y, Yu H, Rozeboom L, et al. LAG-3 Protein Expression in Non-Small Cell Lung Cancer and Its Relationship with PD-1/PD-L1 and Tumor-Infiltrating
Lymphocytes. J Thorac Oncol 2017;12:814-23.

3. National Lung Screening Trial Research Team; Aberle DR, Adams AM, et al. Reduced lung-cancer mortality with low-dose computed tomographic screening. $\mathrm{N}$ Engl J Med 2011;365:395-409.

4. Gould MK, Tang T, Liu IL, et al. Recent Trends in the Identification of Incidental Pulmonary Nodules. Am J Respir Crit Care Med 2015;192:1208-14.

5. LeMense GP, Waller EA, Campbell C, et al. Development and outcomes of a comprehensive multidisciplinary incidental lung nodule and lung cancer screening program. BMC Pulm Med 2020;20:115.

6. Shen KR, Meyers BF, Larner JM, et al. Special treatment issues in lung cancer: ACCP evidencebased clinical practice guidelines (2nd edition). Chest 2007;132:290S-305S.

7. Yildirim E, Kirbas I, Harman A, et al. CT-guided cutting needle lung biopsy using modified coaxial technique: factors effecting risk of complications. Eur J Radiol 2009;70:57-60.

8. Heyer CM, Reichelt S, Peters SA, et al. Computed tomography-navigated transthoracic core biopsy of pulmonary lesions: which factors affect diagnostic yield and complication rates? Acad Radiol 2008;15:1017-26.

9. Gu P, Zhao YZ, Jiang LY, et al. Endobronchial ultrasoundguided transbronchial needle aspiration for staging of lung cancer: a systematic review and meta-analysis. Eur J Cancer 2009;45:1389-96.

10. Adams K, Shah PL, Edmonds L, et al. Test performance of endobronchial ultrasound and transbronchial needle aspiration biopsy for mediastinal staging in patients with lung cancer: systematic review and meta-analysis. Thorax 2009;64:757-62.

11. Huang $Z$, Huang $H$, Ning $Y$, et al. Radial probe endobronchial ultrasound assisted conventional transbronchial needle aspiration in the diagnosis of solitary peribronchial pulmonary lesion located in the segmental bronchi. J Cancer 2019;10:634-42.

12. Wang KP. Staging of bronchogenic carcinoma by bronchoscopy. Chest 1994;106:588-93.

13. Baaklini WA, Reinoso MA, Gorin AB, et al. Diagnostic yield of fiberoptic bronchoscopy in evaluating solitary pulmonary nodules. Chest 2000;117:1049-54.

14. Ost DE, Ernst A, Lei X, et al. Diagnostic Yield and Complications of Bronchoscopy for Peripheral Lung Lesions. Results of the AQuIRE Registry. Am J Respir Crit Care Med 2016;193:68-77.

15. Herth FJ, Eberhardt R, Becker HD, et al. Endobronchial 
ultrasound-guided transbronchial lung biopsy in fluoroscopically invisible solitary pulmonary nodules: a prospective trial. Chest 2006;129:147-50.

16. Ali MS, Trick W, Mba BI, et al. Radial endobronchial ultrasound for the diagnosis of peripheral pulmonary lesions: A systematic review and meta-analysis. Respirology 2017;22:443-53.

17. Torrington KG, Kern JD. The utility of fiberoptic bronchoscopy in the evaluation of the solitary pulmonary nodule. Chest 1993;104:1021-4.

18. Wang Memoli JS, Nietert PJ, Silvestri GA. Metaanalysis of guided bronchoscopy for the evaluation of the pulmonary nodule. Chest 2012;142:385-93.

19. Gildea TR, Mazzone PJ, Karnak D, et al. Electromagnetic navigation diagnostic bronchoscopy: a prospective study. Am J Respir Crit Care Med 2006;174:982-9.

20. Asano F, Shinagawa N, Ishida T, et al. Virtual bronchoscopic navigation combined with ultrathin bronchoscopy. A randomized clinical trial. Am J Respir Crit Care Med 2013;188:327-33.

21. Guisier F, Salaün M, Lachkar S, et al. Molecular analysis of peripheral non-squamous non-small cell lung cancer sampled by radial EBUS. Respirology 2016;21:718-26.

22. Kim H, Park CM, Goo JM, et al. Quantitative Computed Tomography Imaging Biomarkers in the Diagnosis and Management of Lung Cancer. Invest Radiol 2015;50:571-83.

23. Dziedzic D, Peryt A, Szolkowska M, et al. Endobronchial ultrasound-guided transbronchial needle aspiration in the staging of lung cancer patients. SAGE Open Med 2015;3:2050312115610128.

24. Geraghty PR, Kee ST, McFarlane G, et al. CT-guided transthoracic needle aspiration biopsy of pulmonary nodules: needle size and pneumothorax rate. Radiology
2003;229:475-81.

25. Yeow KM, Su IH, Pan KT, et al. Risk factors of pneumothorax and bleeding: multivariate analysis of 660 CT-guided coaxial cutting needle lung biopsies. Chest 2004;126:748-54.

26. Trkanjec JT, Peros-Golubicić T, Grozdek D, et al. The role of transbronchial lung biopsy in the diagnosis of solitary pulmonary nodule. Coll Antropol 2003;27:669-75.

27. de Fenoyl O, Capron F, Lebeau B, et al. Transbronchial biopsy without fluoroscopy: a five year experience in outpatients. Thorax 1989;44:956-9.

28. Steinfort DP, Khor YH, Manser RL, et al. Radial probe endobronchial ultrasound for the diagnosis of peripheral lung cancer: systematic review and meta-analysis. Eur Respir J 2011;37:902-10.

29. Fuso L, Varone F, Magnini D, et al. Role of ultrasoundguided transbronchial biopsy in the diagnosis of peripheral pulmonary lesions. Lung Cancer 2013;81:60-4.

30. Jacomelli M, Demarzo SE, Cardoso PF, et al. Radial-probe EBUS for the diagnosis of peripheral pulmonary lesions. J Bras Pneumol 2016;42:248-53.

31. Almeida FA, Salam S, Mehta AC, et al. Sampling Utility of the Convex Probe Endobronchial Ultrasound Visible Intrapulmonary Lesion. J Bronchology Interv Pulmonol 2018;25:290-9.

32. Yu KL, Tsai TH, Ho CC, et al. The value of radial endobronchial ultrasound-guided bronchial brushing in peripheral non-squamous non-small cell lung cancer. Sci Rep 2018;8:5837.

33. Sánchez-Font A, Chalela R, Martín-Ontiyuelo C, et al. Molecular analysis of peripheral lung adenocarcinoma in brush cytology obtained by EBUS plus fluoroscopy-guided bronchoscopy. Cancer Cytopathol 2018;126:860-71.
Cite this article as: Song N, Yang L, Wang H, Jiang L, Zhao L, Colella S, Jagan N, Almeida FA, Wu L, Gu Y, He Y. Radial endobronchial ultrasound-assisted transbronchial needle aspiration for pulmonary peripheral lesions in the segmental bronchi adjacent to the central airway. Transl Lung Cancer Res 2021;10(6):2625-2632. doi: 10.21037/tlcr-21-490 


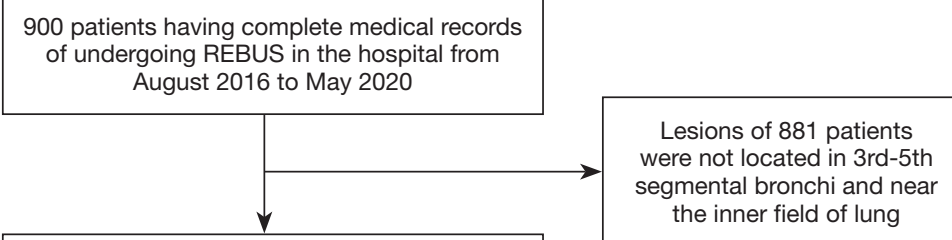

19 patients were eligible for this current study

Figure S1 Flow diagram for including patient. 